\title{
One and two-dimensional quantum models: quenches and the scaling of irreversible entropy
}

\author{
Shraddha Sharma ${ }^{1}$ and Amit Dutta ${ }^{1}$ \\ ${ }^{1}$ Department of Physics, Indian Institute of Technology, 208016, Kanpur
}

\begin{abstract}
Using the scaling relation of the ground state quantum fidelity, we propose the most generic scaling relations of the irreversible work (the residual energy) of a closed quantum system at absolute zero temperature when one of the parameters of its Hamiltonian is suddenly changed; we consider two extreme limits namely, the heat susceptibility limit and the thermodynamic limit. It is then argued that the irreversible entropy generated for a thermal quench at low enough temperatures when the system is initially in a Gibbs state, is likely to show a similar scaling behavior. To illustrate this proposition, we consider zero-temperature and thermal quenches in one and two-dimensional Dirac Hamiltonians where the exact estimation of the irreversible work and the irreversible entropy is indeed possible. Exploiting these exact results, we then establish: (i) the irreversible work at zero temperature indeed shows an appropriate scaling in the thermodynamic limit; (ii) the scaling of the irreversible work in the 1D Dirac model at zero-temperature shows logarithmic corrections to the scaling which is a signature of a marginal situation. (iii) Furthermore, remarkably the logarithmic corrections do indeed appear in the scaling of the entropy generated if temperature is low enough while disappears for high temperatures. For the 2D model, no such logarithmic correction is found to appear.
\end{abstract}

PACS numbers:

\section{INTRODUCTION}

Studying emergent thermodynamics following a quench of a closed quantum system initially in equilibrium with a heat reservoir has attracted the attention of the scientists in recent years ${ }^{1-4}$. These studies are also important from the viewpoint of non-equilibrium fluctuation theorems ${ }^{1,2,5,6}$ and quantum Jarzynski equality ${ }^{7}$. Treating a sudden quench (i.e., a change) of a parameter of the Hamiltonian as a thermodynamic transformation and decoupling the system from the bath so that the evolution of the system following a quench is perfectly unitary, a generalized second law (or Clausius inequality) for an isolated quantum system has been posited with the entropy generated in the process being defined in terms of the irreversible work done in the process of quenching $3,4,8$. These studies have been generalized to the context of Bures metric $\frac{9}{\underline{-}}$ and Uhlmann fidelity ${ }^{10}$, single qubit interferrometry ${ }^{11}$, open quantum systems ${ }^{12}$ and inner friction of quantum thermodynamic processes 13

In the above studies, the quantum system is initially prepared in a mixed state in equilibrium with a heat bath which is then decoupled and the system is subjected to a sudden change (or a slow ramp dictated by a protocol described by a unitary operator $\hat{U}$ ) of one of the parameters. Due to the stochastic nature of the work done for a finite system (which is not an observable ${ }^{2}$ ), one makes resort to the characteristic cumulant-generating function. To elaborate, let us consider a system described by the Hamiltonian $H(\lambda)$ which is at the quantum critical point (QCP) at $\lambda=0^{14-16}$; we assume that at an initial time $t=0$ the system is kept in contact with a heat bath at an inverse temperature $\beta$ with an initial value of $\lambda=\lambda_{0}$. The system is then decoupled from the bath and the parameter $\lambda$ is tuned from the initial value $\lambda_{0}$ to the final value $\lambda_{f}=\lambda_{0}+\delta$, so that the subsequent temporal evolution of the system is entirely dictated by the unitary operator $\hat{U}$. Implementing the analytical continuation $s \rightarrow-i t$, one defines the characteristic function

$$
G(s)=\operatorname{Tr}\left[\hat{U}^{\dagger} e^{s H\left(\lambda_{f}\right)} \hat{U} e^{-s H\left(\lambda_{0}\right)} \rho_{0}\left(\lambda_{0}\right)\right] ;
$$

here, $\rho_{0}\left(\lambda_{0}\right)=e^{-\beta H\left(\lambda_{0}\right)} / Z\left(\lambda_{0}\right)$ is the density matrix characterizing the initial Gibbs state and $Z\left(\lambda_{0}\right)$ is the corresponding partition function. For an instantaneous quench from $\lambda_{0}$ to $\lambda_{f}, G(s)$ takes a simpler form:

$$
G(s)=\operatorname{Tr}\left[e^{s H\left(\lambda_{f}\right)} e^{-s H\left(\lambda_{0}\right)} \rho_{0}\left(\lambda_{0}\right)\right] .
$$

At this juncture, it should be noted that the characteristic function $G(s)$ closely resembles the mixed state Loschmidt echo $\frac{17}{}$ which has been extensively studied in the context of zero-temperature non-equibrium dynamics of quantum critical systems ${ }^{18}-22$ and associated dynamical phase transition ${ }^{23}$. Moreover, $G(s)$ is related (through the inverse Fourier or Laplace transform) to the distribution function $P(W)$ of work $(W)$, which is usually characterized by an edge singularity at zerotemperature $24-26$.

Focussing on the sudden quenching case, the average work performed is defined through the first cumulant of the characteristic function, as shown:

$$
\langle W\rangle=d G(s) /\left.d s\right|_{s=0}=\operatorname{Tr}\left(H\left(\lambda_{f}\right) \rho_{0}\right)-\operatorname{Tr}\left(H\left(\lambda_{0}\right) \rho_{0}\right) .
$$

To arrive at the generalized second law, we note that for a non-equilibrium process, the average work $\langle W\rangle$ always exceeds the free energy difference $(\Delta F)$ between the initial and final equilibrium states (both with inverse temperature $\beta$ ) with parameters $\lambda_{0}$ and $\lambda_{f}$, respectively. 
One can define the irreversible work through the difference $W_{\text {irr }}^{T}=\langle W\rangle-\Delta F$. The heat exchange with the bath for a closed quantum system during the evolution being zero, the irreversible entropy generated in the process satisfies the relation $\Delta S_{\mathrm{irr}}=\beta W_{\mathrm{irr}}^{T}$. In the subsequent discussion, we shall drop the superscript $T$ and use $W_{\text {irr }}$ to denote the finite temperature irreversible work.

For a sudden quench of magnitude $\delta$ at zerotemperature, when $\Delta F \rightarrow\left(E_{g}\left(\lambda_{f}\right)-E_{g}\left(\lambda_{0}\right)\right)$, Eq. (3) reduces to

$$
W_{\text {irr }}^{T=0}=\left\langle\psi_{g}\left(\lambda_{0}\right)\left|H\left(\lambda_{f}\right)\right| \psi_{g}\left(\lambda_{0}\right)\right\rangle-E_{g}\left(\lambda_{f}\right) ;
$$

here, $\left|\psi_{g}\left(\lambda_{0}\right)\right\rangle$ is the ground state wave function corresponding to the initial Hamiltonian $H\left(\lambda_{0}\right)$ and $E_{g}\left(\lambda_{i}\right)\left(E_{g}\left(\lambda_{f}\right)\right)$ is the ground state energy of $H\left(\lambda_{i}\right)$ $\left(H\left(\lambda_{f}\right)\right)$. Clearly, $W_{\text {irr }}^{T=0}$ is the same as the residual energy (excess energy above the ground state) and hence should follow an identical scaling relation for a sudden quench obtained using an adiabatic perturbation theory as reported in the literature $\underline{16,27-29}$. It is noteworthy that very recently, the quantity $W_{\text {irr }}^{T=0}$ has been studied establishing a connection to the heat susceptibility (for $\delta \rightarrow 0$ ) in the context of quantum latency which detects the order of a quantum phase transition (QPT) ${ }^{30}$.

Let us now ask the question how does the proximity to a QCP influence the behavior of $\Delta S_{\text {irr }}$ and explore its scaling behavior in terms of the deviation from the QCP $(\lambda)$, the magnitude of the quench $\delta=\lambda_{f}-\lambda_{0}$ and the system size $L$. In Ref. [4], it was shown how the form of $G(s)$ given in Eq. (11) can be exploited to establish the Tasaki-Crooks relation and the Jarzynski equality for a closed quantum system. Taking the example of a 1D quantum Ising model, it has also been shown that when $\lambda$ is varied keeping $\delta$ small and $L$ finite, $\Delta S_{\text {irr }}$ shows a sharp peak at the QCP if $\beta$ is large, while on the contrary in the high temperature situation $(\beta \rightarrow 0), \Delta S_{\text {irr }}$ gets broadened. One therefore concludes that the peak in $\Delta S_{\text {irr }}$ is an indicator of a QPT even at a low but finite temperature.

In this paper, we shall first consider $W_{\mathrm{irr}}^{T=0}$ and inspired by the scaling of the quantum fidelity explore its scaling behavior for a sudden quench of magnitude $\delta$ in the vicinity of a QCP characterized by the associated quantum critical exponents in different limits $\lambda, \delta$ and $L$; both the situations when $\delta$ can be treated as a perturbation ${ }^{30}$ or it is a scaling variable will be probed. We shall then address the question whether $\Delta S_{\text {irr }}$ generated for such a quench exhibit similar scaling relations. To the best of our knowledge, our work is the first attempt that generalizes the derivation of $\Delta S_{\text {irr }}$ to a higher dimensional system where an exact analytical calculation is possible. The exact analytical form enables us to derive the scaling of $W_{\text {irr }}^{T=0}$ and $\Delta S_{\text {irr }}$ when $\delta \rightarrow 0$ and also for finite $\delta$.

The paper is organized in the following manner: the scaling relation of $W_{\mathrm{irr}}^{T=0}$ and $\Delta S_{\mathrm{irr}}$ are proposed in Sec. III In Sec. III we shall invoke upon the one dimensional (1D) and two dimensional (2D) Dirac Hamiltonians to illustrate the scaling relations proposed earlier. The advantage of using a Dirac Hamiltonian is the inherent $2 \times 2$ nature of the same rendering it integrable in all dimensions; in addition, 1D Dirac Hamiltonian, as we shall show below, provides an ideal example of a marginal situation where we find the signature of logarithmic corrections to the scaling of $W_{\text {irr }}^{T=0}$ which persist in $\Delta S_{\text {irr }}$ at low but finite temperatures and disappears in the $2 \mathrm{D}$ case. In the $2 \mathrm{D}$ case, in contrast to the $1 \mathrm{D}$ case, $\Delta S_{\text {irr }}$ does not show a sharp peak at the QCP and hence can not be treated as an ideal indicator of a QPT. Concluding comments are presented in Sec. IV]

\section{SCALING RELATIONS OF $W_{\mathrm{irr}}$ AND $\Delta S_{\mathrm{irr}}$}

Before embarking upon the study of $\Delta S_{\text {irr }}$ at finite temperature, we piece together the scaling of the defect density and the residual energy at zero temperature following a sudden quench considering different limits: we first consider the situation when $L$ is the largest length scale of the problem which implies $L \gg \delta^{-\nu}, \lambda^{-\nu}$, where $\nu$ is the correlation length exponent associated with the QCP; clearly, in this case the parameter $\delta$ is finite and hence can not be treated as a perturbation. We shall arrive at the scaling relation of $W_{\mathrm{irr}}^{T=0}$ using heuristic arguments inspired by the scaling of the ground state quantum fidelity $\underline{31}-\underline{33}, F(\lambda, \delta)=\left|\left\langle\psi_{g}\left(\lambda_{0}\right) \mid \psi_{g}\left(\lambda_{0}+\delta\right)\right\rangle\right|$ given by $\underline{34}$

$$
\ln F=-L^{d}|\delta|^{\nu d} \mathcal{F}\left(\frac{\lambda}{\delta}\right)
$$

where $\mathcal{F}$ is the corresponding scaling function, $\nu$ is the correlation length exponent associated with the QCP and $d$ is the spatial dimension; $L$ being the largest length scale of the problem, this limit is known as the thermodynamic limit. Let us first consider the situation, $\lambda \ll \delta$, which means $\delta^{-\nu} \ll \lambda^{-\nu}$, i.e., the correlation length $\xi \sim \lambda^{-\nu}$ is larger than the length scale associated with the parameter $\delta$, so effectively the system is close to the QCP. Using the finite size scaling argument $\frac{35}{2}$, we expect $\ln F$ to scale with $\delta$. Demanding that right hand side of Eq. (5) is dimensionless, we find a characteristic momentum scale $\hat{k} \sim \delta^{\nu}$ (since we know that $\delta^{-\nu}$ has the dimension of length). Using the phase space argument, one then finds that the defect (or quasi-particle) density $(n)$ scales as $\hat{k}^{d} \sim \delta^{\nu d}$. (For $\nu=d=1$, it has been proved 36 that one has $n \sim|\delta|$.) We note that as predicted in Ref. [27], the scaling of defect density is different from the residual energy for a sudden quench in the vicinity of the QCP; in this case, each excitation carries energy $\delta^{\nu z}$, where $z$ is the dynamical exponent, resulting in the scaling $W_{\text {irr }}^{T=0} \sim \delta^{\nu(d+z) \underline{27}, 37}$.

We now consider the other limit, when $\lambda \gg \delta$, (implying $\lambda^{-\nu} \ll \delta^{-\nu}$, i.e., away from the QCP), $\lambda$ is expected to play the role of the scaling variable. The quantum fidelity defined in Eq. (5) is expected to scale as $\ln F=$ 
$-L^{d} \delta^{2} \lambda^{\nu d-2}$; in a similar spirit identifying the characteristic momentum scale, it is straightforward to arrive at the scaling of the defect density as $n \sim \hat{k}^{d} \sim \delta^{2} \lambda^{\nu d-2}$ which has already been established for the quantum Ising $\operatorname{case}^{36}(\nu=d=z=1)$ enabling us to arrive at the scaling relation, $W_{\text {irr }}^{T=0} \sim \delta^{2} \lambda^{\nu d+\nu z-2}=\delta^{2} \lambda^{-\alpha}$ where $\alpha$ is the corresponding "specific heat" exponent.

We have illustrated above how the scaling of fidelity can lead to the scaling of $W_{\text {irr }}^{T=0}$ in the thermodynamic limit. Concerning the scaling of fidelity, it is well-known that one can switch from the thermodynamic limit to the fidelity susceptibility $\left(\chi_{F}(\lambda)\right)$ limit $\underline{27}, \underline{37} \underline{\underline{39}}$ (where the notion of the fidelity susceptibility is meaningful) when $\delta^{-\nu} \gg L$. Continuing along the same line of arguments, one can define the heat susceptibility $\chi_{E} \frac{27}{}$ such that $W_{\text {irr }}^{T=0} \sim \delta^{2} \chi_{E}$, when $\delta^{-\nu}$ (i.e, $\delta \rightarrow 0$ ) is the largest length scale. In this limit, one expects a scaling $W_{\text {irr }}^{T=0} \sim \delta^{2} \lambda^{-\alpha}$ away from the QCP and on the other hand, close to the QCP, $L$ plays the role of the scaling variable in lieu of $\lambda$. To summarize, we have:

Heat Susceptibility limit: $\delta^{-\nu}$ is the largest length scale and $\delta(\rightarrow 0)$ can be treated as a perturbation

$$
\begin{aligned}
W_{\mathrm{irr}}^{T=0} / L & \sim \delta^{2} \lambda^{\nu(d+z)-2} \quad \delta^{-\nu}>L>\lambda^{-\nu} \\
& \sim \delta^{2} L^{2 / \nu-(d+z)} \quad \delta^{-\nu}>\lambda^{-\nu}>L .
\end{aligned}
$$

The crossover between the limits close and away from the QCP occurs when $L \sim \lambda^{-\nu}$.

Thermodynamic limit: $L$ is the largest length scale:

$$
\begin{array}{rlrl}
W_{\mathrm{irr}}^{T=0} / L & \sim \delta^{2} \lambda^{\nu(d+z)-2} & & L>\delta^{-\nu}>\lambda^{-\nu} \\
& \sim \delta^{\nu(d+z)} & L>\lambda^{-\nu}>\delta^{-\nu} .
\end{array}
$$

Similarly here the crossover from one scaling to the other occurs when $\lambda \sim \delta$. As explained above the crossover from the thermodynamic limit to the susceptibility limit occurs when $\delta^{-\nu} \sim L$. At this point, it would be instructive to note that what we present above happens to be the most general scaling relations of $W_{\text {irr }}^{T=0}$. If the system is precisely at the QCP $\left(\lambda^{-\nu} \rightarrow \infty\right)$, one arrives at the scaling relations (with $L$ or $\delta$ ) proposed in Ref. 27] by setting $\lambda=\delta$ in the scaling relations and switching $\lambda$ and $\delta$ in the corresponding conditions in Eqs. (6).

We would like to emphasize here that when the combination $\nu(d+z)$ exceeds 2 , the scaling of $W_{\text {irr }}$ is nonuniversal. In the marginal case when $\nu(d+z)=2$, one encounters additional logarithmic corrections to the scaling and one needs to introduce an upper cut-off in the momentum scale $k_{\max }$ as we shall illustrate below for the 1D Dirac model.

We shall now proceed to analyze the scaling of $\Delta S_{\text {irr }}$ generated following a sudden change of $\lambda$ by an amount $\delta$ while the system was initially in thermal equilibrium with a heat bath at a temperature $T$. Question we raise is that wether $\Delta S_{\mathrm{irr}}=\beta W_{\mathrm{irr}}$, is likely to show a similar scaling relation for such a thermal quench. Using a perturbative expansion of the free energy (corresponding to the final parameter) valid in the limit $\delta \rightarrow 0$, a scaling $\Delta S_{\text {irr }} \sim \delta^{2} \lambda^{-\alpha}$, has been proposed ${ }^{8}$; obviously, a similar expansion is not possible in other limits mentioned in Eqs. (6) and (7). In the finite temperature case, the system is initially in a mixed state. Nevertheless, for low enough temperatures the ground state of the Hamiltonian is maximally populated and one would therefore expect $\Delta S_{\text {irr }}$ to satisfy a similar scaling as $W_{\text {irr }}^{T=0}$; on the contrary, these scaling relations should disappear at higher temperatures when the initial state deviates significantly from the ground state. Our aim here is to show that this indeed is the case in all the limits not only for $\delta \rightarrow 0$. Furthermore, we shall illustrate how to arrive at these scaling relations in both the susceptibility limit and the thermodynamic limit for an integrable Hamiltonian. Finally, the question whether at a low temperature $\Delta S_{\text {irr }}$ always shows a sharp peak at the QCP will be addressed.

\section{SCALING IN DIRAC HAMILTONIANS AND ROLE OF MARGINALITY}

In this section, we shall derive the scaling relations of $W_{\text {irr }}^{T=0}$ and address the issues raised in the previous section concerning the scaling of $\Delta S_{\text {irr }}$ using the example of $1 \mathrm{D}$ and 2D Dirac Hamiltonians which are very important from theoretical point of view in quantum condensed matter systems. For example, we take a $2 \mathrm{D}$ massive Dirac Hamiltonian $\hat{H}_{D}=\int d \mathbf{x} \widehat{\Psi}^{\dagger}(\mathbf{x})\left[m \hat{\sigma}_{z}-\right.$ $\left.i \hbar v_{F}\left(\hat{\sigma}_{x} \partial_{x}+\hat{\sigma}_{y} \partial_{y}\right)\right] \widehat{\Psi}(\mathbf{x})$, where $\widehat{\Psi}(\mathbf{x})$ is a two-component spinor field operator describing, the effective low-energy degrees of freedom for electrons on a honeycomb lattice with unequal sublattice potentials around a single Dirac point (i.e., single valley graphene Hamiltonian) with Fermi velocity $v_{F}$ (set equal to unity below) $\stackrel{40}{\underline{n}}$. Variations of this model are ubiquitous in the field of topological insulators 11,42 . The edge states in a $2 \mathrm{D}$ topological insulator are described by an effective 1D Dirac Hamiltonian, whereas its bulk states are described by a 2D Dirac Hamiltonian and the QCP separating the gapped to gapless phases is a 2D massless Dirac point. These models have turned out to be immensely useful in the studies of the Kibble-Zurek scaling $\underline{43}$, sudden quenches $\underline{44}, 45$, fidelity susceptibility and thermodynamic fidelity $\underline{46}$, Loschmidt echo $\frac{47}{2}$ and periodic steady state reached through a sinusoidal variation of the mass term ${ }^{48}$. Considering the massive 2D Dirac Hamiltonian one can rescale the units appropriately to obtain the $2 \times 2$ Hamiltonian describing the system close to a single valley as,

$$
H_{2 D}(m)=\left(\begin{array}{cc}
m & k_{x}-i k_{y} \\
k_{x}+i k_{y} & -m
\end{array}\right)
$$

here, $m$ is the Dirac mass and the vector $\vec{k}$ is measured with respect to the corners of the Brillouin zone. 
The scaling relations obtained for the $1 \mathrm{D}$ massive Dirac Hamiltonian:

$$
H_{1 D}(m)=\left(\begin{array}{cc}
m & k \\
k & -m
\end{array}\right)
$$

are to be compared with the corresponding $2 \mathrm{D}$ case. Both the 1D and 2D models exhibit a QCP where the mass term $m$ vanishes and the dispersion at the QCP is linear. These QCPs are then characterized by the critical exponents $\nu=z=1$. In the sense of universality $H_{1 D}$ is identical to a 1D quantum Ising chain; the later can be recast to the form given in Eq. (9) with $m$ being the deviation of the transverse field from its quantum critical value. The $1 \mathrm{D}$ case is conspicuous in the sense that we have $\nu(d+z)=2$. As a result, one expects a logarithmic correction to the scaling of $W_{\mathrm{irr}}^{T=0}$ and remarkably this logarithmic scaling persists in $\Delta S_{\text {irr }}$ even at low temperature. On the contrary, no such logarithmic singularity is observed in the $2 \mathrm{D}$ case.

Using Hamiltonians (8) and (9), we shall consider a sudden quench of the mass term $m$ (which will still be denoted by the parameter $\lambda$ for consistency) from an initial value $\lambda$ to a final value $\lambda+\delta$ and calculate the exact analytical expressions for $W_{\text {irr }}^{T=0}$ and $\Delta S_{\text {irr }}$; this would in turn lead to the scaling relations of these quantities both in the susceptibility as well as the thermodynamic limit. by

Using the eigenstates of 1-D Dirac Hamiltonian given

$\left|\psi_{k}{ }^{ \pm}\left(\lambda_{0}\right)\right\rangle_{1 D}=\frac{k}{\sqrt{\left(\lambda_{0}-\sqrt{k^{2}+\lambda_{0}^{2}}\right)^{2}+k^{2}}}\left(\frac{\lambda_{0}-\sqrt{k^{2}+\lambda_{0}^{2}}}{k}\right)$,

with corresponding eigen-energies $E\left(\lambda_{0}\right)_{1 D}=$ $\pm \sqrt{k^{2}+\lambda_{0}^{2}}$ in Eq. (44), the expression for $W_{\mathrm{irr}}^{T=0}$ can be derived in a closed form

$$
\begin{aligned}
W_{\operatorname{irr} 1 D}^{T=0} & =\frac{1}{2}\left[k_{\max } \sqrt{k_{\max }^{2}+(\lambda+\delta)^{2}}-k_{\max } \sqrt{k_{\max }^{2}+\lambda^{2}}+\left(\frac{2 \pi}{L}\right) \sqrt{\left(\frac{2 \pi}{L}\right)^{2}+\lambda^{2}}\right. \\
& -\left(\frac{2 \pi}{L}\right) \sqrt{\left(\frac{2 \pi}{L}\right)^{2}+(\lambda+\delta)^{2}}+(\lambda+\delta)^{2} \log \left(\frac{k_{\max }+\sqrt{k_{\max }^{2}+(\lambda+\delta)^{2}}}{\frac{2 \pi}{L}+\sqrt{\left(\frac{2 \pi}{L}\right)^{2}+(\lambda+\delta)^{2}}}\right) \\
& \left.+\lambda(\lambda+2 \delta) \log \left(\frac{\frac{2 \pi}{L}+\sqrt{\left(\frac{2 \pi}{L}\right)^{2}+\lambda^{2}}}{k_{\max }+\sqrt{k_{\max }^{2}+(\lambda+\delta)^{2}}}\right)\right] .
\end{aligned}
$$

Here, we have converted the sum over $k$ to the integral using a periodic boundary condition with the step size $2 \pi / L ; k_{\max }$ plays the role of the upper cut off in the momentum scale, i.e., $k_{\max }^{-1}$ which is the shortest length scale of the problem (i.e., the lattice spacing). This arises because of the fact that the integral in Eq. (111) can not be extended to $k \rightarrow \infty$ and to avoid divergence one needs to introduce a cut-off. Furthermore, as is evident in the expression in Eq. (11), the presence of $k_{\max }$ in fact renders the argument of logarithm dimensionless. Similarly, one can derive an exact analytical form of the $W_{\text {irr }}^{T=0}$ in the $2 \mathrm{D}$ case also. In Fig. (1), we show the variation of $W_{\text {irr }}^{T=0}$ as a function of $\lambda$ systematically in different limits. We note that when plotted on the same scale, there is a significant peak at the QCP in the thermodynamic limit while the peak almost disappears in the susceptibility limit.

Using the exact form of $W_{\text {irr }}^{T=0}$ as given in Eq. (11), one can now derive the scaling behavior of the same in different limits: in the susceptibility limit $\delta^{-1}$ is the largest length scale that never appears in the scaling. In this limit, we then find:

$$
\begin{aligned}
W_{\mathrm{irr}}^{T=0} / L & \sim \delta^{2} \log \left(2 k_{\max } / \lambda\right) ; \delta^{-\nu}>L>\lambda^{-\nu}>k_{\max }^{-1} \\
& \sim \delta^{2} \log \left(L k_{\max } / 2 \pi\right) ; \delta^{-\nu}>\lambda^{-\nu}>L>k_{\max }^{-1}
\end{aligned}
$$

On the other hand, in the thermodynamic limit where $L$ is the largest length scale, using Eq. (11), we get

$$
\begin{aligned}
W_{\mathrm{irr}}^{T=0} / L & \sim \delta^{2} \log \left(2 k_{\max } / \lambda\right) ; L>\delta^{-\nu}>\lambda^{-\nu}>k_{\max }^{-1} \\
& \sim \delta^{2} \log \left(2 k_{\max } / \delta\right) ; L>\lambda^{-\nu}>\delta^{-\nu}>k_{\max }^{-1}
\end{aligned}
$$

The scaling given in Eq. (14) might look identical to that given in Eq. (12); however, we would like to emphasize that these two relations are derived from the exact expression in the different limits. These scaling relations in the $1 \mathrm{D}$ and 2D cases are illustrated in Figs. (2) and (3) choosing the appropriate values of the parameters in 


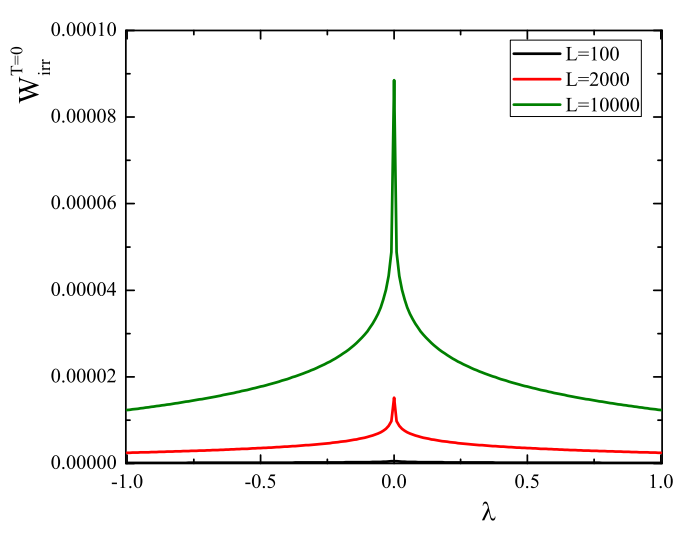

FIG. 1: The plot of $W_{\text {irr }}^{T=0}$ in different limits showing peak at the $\mathrm{QCP}(\lambda=0)$ for a 1D Dirac Hamiltonian There is no visible peak in the susceptibility limit $(L=100$, bottom most) and there is a sharp peak in the thermodynamic limit ( $L=10000$, topmost). In the intermediate case, $L=2000$, there is a significant peak. Here, $\delta=0.001$ and $k_{\max }=2 \pi$ in all the cases.

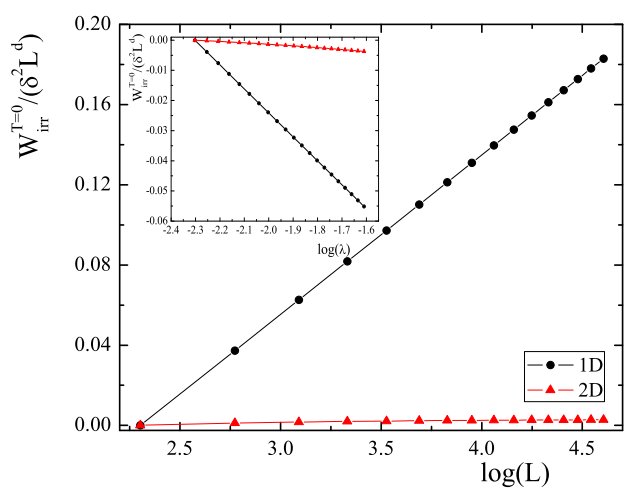

FIG. 2: The logarithmic dependence with $\lambda$ (away from the QCP) and $L$ (close to QCP) for 1D Dirac model can be seen which is clearly not present in the $2 \mathrm{D}$ case in the susceptibility limit. The main figure verifies the logarithmic scaling with $L$ when $\lambda=0.01$. Inset shows the logarithmic dependence with $\lambda$ for $L=100$ in $1 \mathrm{D}$ case and no such dependence for 2D case with $L_{x}=L_{y}=100$. In the main figure as well as in the inset $\delta=0.001$ while $k_{\max }=\pi$.

different limits. We find that the leading scaling behavior of $W_{\text {irr }}^{T=0}$ is given by $\delta^{2}$; in the $1 \mathrm{D}$ case, there exist additional logarithmic corrections to the scaling with a scaling variable appropriate in the limit under consideration. No such logarithmic correction is seen for the 2D case which can be established using a similar exact analytical form of $W_{\text {irr }}^{T=0}$. Remarkably as we claimed earlier, in Fig. (2), the parameter $\delta$ indeed appears as a scaling variable in the thermodynamic limit when the system is close to the QCP; this is one of our most important find-

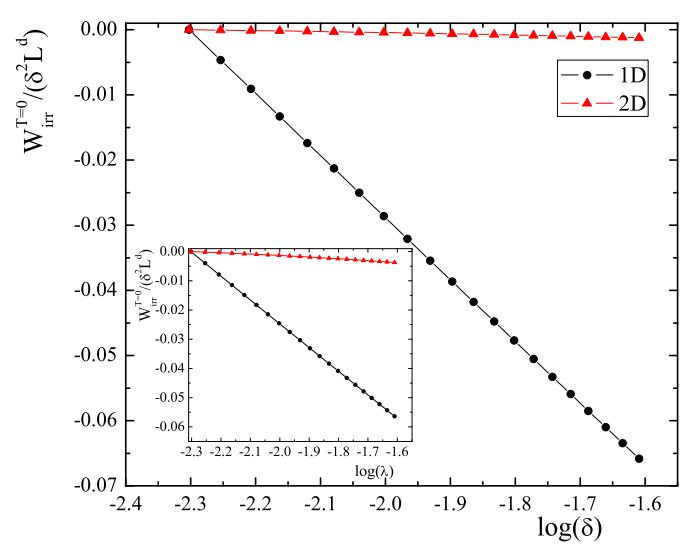

FIG. 3: $\quad W_{\text {irr }}^{T=0} /\left(\delta^{2} L^{d}\right)$ for $1 \mathrm{D}$ and 2D Dirac Hamiltonian verifies the scaling proposed in the main text, showing a logarithmic scaling in the marginal situation (i.e. 1D Dirac model) which is absent in the $2 \mathrm{D}$ case. The main figure clearly confirms the scaling with $\delta$ setting $\lambda=0.01$. Inset on the other hand shows the expected scaling away from the QCP in the thermodynamic limit with $\delta=0.01$. The parameters for both the figures (main and inset) are $L=1000$ and $k_{\max }=\pi$.

ings which was never reported before.

Let us proceed to the finite temperature situation, and ask the question whether similar logarithmic corrections are present in $\Delta S_{\text {irr }}$. The inherent $2 \times 2$ nature of the Dirac Hamiltonians enables us to calculate an exact expression for $\Delta S_{\text {irr }}$ also. For a single $k$ mode, one finds

$$
\begin{aligned}
\Delta S_{\mathrm{irr} k} & =\beta\left(E_{k}\left(\lambda_{0}\right)+E_{k}\left(\lambda_{f}\right)\left(1-2\left|\left\langle\psi_{k}^{-}\left(\lambda_{0}\right) \mid \psi_{k}^{-}\left(\lambda_{f}\right)\right\rangle\right|^{2}\right)\right. \\
& \times \tanh \left(\frac{\beta E_{k}\left(\lambda_{0}\right)}{2}\right)+2 \log \frac{\cosh \left(\beta E_{k}\left(\lambda_{f}\right)\right)}{\cosh \left(\beta E_{k}\left(\lambda_{0}\right)\right)} \cdot(16)
\end{aligned}
$$

Integrating the expression given in Eq. (16) over all the momenta modes, one can derive the exact analytical forms of $\Delta S_{\mathrm{irr}}$ both in $1 \mathrm{D}$ and $2 \mathrm{D}$ models. Analyzing those exact expressions, in the appropriate range of parameter values we can extract the scaling behavior of $\Delta S_{\text {irr }}$ which we have illustrated in Figs. (44) and (5). What is remarkable is that even though the leading behavior of the scaling of $\Delta S_{\text {irr }}$ is still given by $\delta^{2}$ for both 1D and 2D Dirac models, the logarithmic correction to the scaling persists in the $1 \mathrm{D}$ case for relatively low temperature while it approaches the $2 \mathrm{D}$ case (when there is no logarithmic correction even at $T=0$ ) as thermal fluctuations increase. This establishes our claim that the proposed scaling of $W_{\mathrm{irr}}^{T=0}$ indeed manifests in $\Delta S_{\mathrm{irr}}$ at low temperature.

Finally, we ask the question whether $\Delta S_{\text {irr }}$ does always exhibit a sharp peak at the QCP when plotted as a function of the tuning parameter $\lambda$ (see Fig. (66)); in 1D case indeed it does which resembles the observation reported in Fig. (1) of the Ref. [4]. However, we would like to draw attention to the symmetric nature of $\Delta S_{\text {irr }}$ on either side of the QCP unlike the transverse Ising case. On 


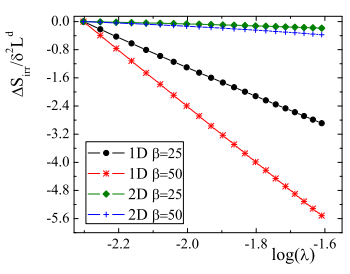

(a) $\Delta S_{\text {irr }} / L^{d}$ shows a logarithmic scaling with $\lambda$ in 1D Dirac with $L=100$; the slope approaches the $2 \mathrm{D}$ case with increasing temperature where there is no logarithmic scaling.

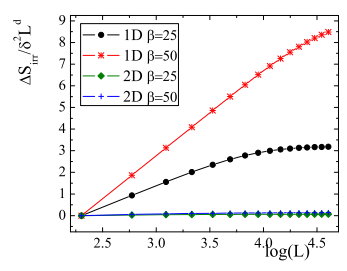

(b) The same for the dependence with $L$ with $\lambda=0.01$.
FIG. 4: We show that in the susceptibility limit, the logarithmic scaling of $\Delta S_{\mathrm{irr}} / L^{d}$ persists for low-temperature in the 1D Dirac case and its comparison with that of 2D Dirac where no such scaling is expected. We have taken $\delta=0.001$ and $k_{\max }=\pi$.

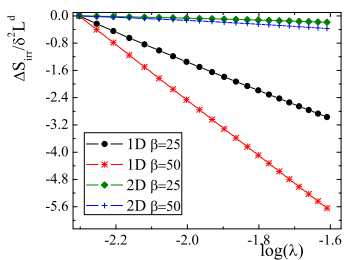

(a) The scaling of $\Delta S_{\mathrm{irr}} / L^{d}$ in the thermodynamic limit, showing clear log dependence in 1D Dirac case which is clearly not observed in $2 \mathrm{D}$ Dirac case with $\delta=0.01$.

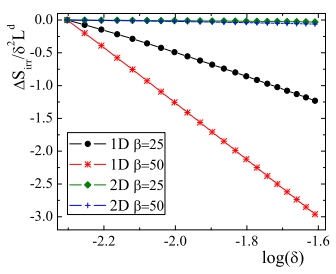

(b) Scaling of $\Delta S_{\mathrm{irr}} / L^{d}$ with $\delta$ shows a clear marginal behavior as a function of $\delta$ (close to QCP) for 1D Dirac with $\lambda=0.01$.
FIG. 5: For $1 \mathrm{D}$ case there is a logarithmic correction to the scaling of $\Delta S_{\text {irr }}$ even in the thermodynamic limit which disappears at high temperatures when the behavior is identical to that in the $2 \mathrm{D}$ case. Here, $L=1000$ and $k_{\max }=\pi$.

the contrary in the $2 \mathrm{D}$ case, there is no sharp peak even at sufficiently low temperature. This leads us to the conclusion that above the marginal situation, $\Delta S_{\text {irr }}$ fails to be a good indicator of a QPT occurring at $T=0$.

\section{CONCLUDING COMMENTS}

In this paper, we have studied quantum and thermal quenches of a closed quantum system; in particular, our focus has been restricted to the case when a parameter $\lambda$ of the Hamiltonian which is close to its QCP (at $\lambda=$ 0 ) is suddenly changed by an amount $\delta$. Based on the three length scales of the problem, namely $\lambda^{-\nu}, \delta^{-\nu}$ and the system size $L$, we have predicted the existence of two scaling regions, namely the susceptibility limit and the thermodynamic limit. Inspired by the scaling of the ground state fidelity in these limits, we have proposed the scaling of $W_{\mathrm{irr}}^{T=0}$ in these limits both close to the QCP and away from the QCP; furthermore, a logarithmic correction with the parameter $\delta$ appears in the scaling close to the QCP establishing that in the thermodynamic limit $\delta$ obviously plays the role of a scaling variable.

We then asked the question whether these scaling relations survive in the case of a sudden quench in which the initial state is a mixed state in thermal equilibrium with a heat bath and manifest in $\Delta S_{\text {irr }}$. To address this question in a transparent way, we have used 1D and 2D Dirac Hamiltonians where arriving at the exact expressions for both $W_{\text {irr }}^{T=0}$ and $\Delta S_{\text {irr }}$ is indeed possible. These exact expressions are then analyzed to derive the scaling relations in different limits. Remarkably, our study establishes the logarithmic correction (which is a signature of marginality) appearing in the scaling of $W_{\text {irr }}^{T=0}$ for 1D indeed survives in the scaling of $\Delta S_{\text {irr }}$ for low enough temperatures. There is no trace of any logarithmic corrections in the $2 \mathrm{D}$ case where the sharp peak in $\Delta S_{\text {irr }}$ plotted as a function of $\lambda$ also get broadened thereby diminishing its usefulness as an ideal detector of a QPT.

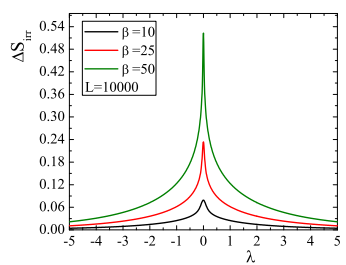

(a) $\Delta S_{\text {irr }}$ showing peak at the QCP $(\lambda=0)$ for a 1D Dirac Hamiltonian. The peak height changes with the temperature keeping $\delta$ fixed to 0.01

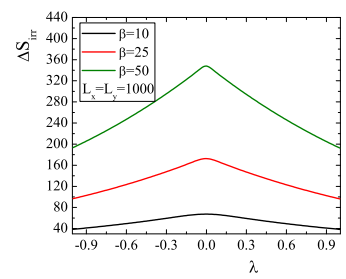

(b) $\Delta S_{\text {irr }}$ versus $\lambda$ shows peak at the QCP for 2D Dirac changing $\beta$.
Hamiltonian for $\delta=0.01$ and

FIG. 6: The figure above shows $\Delta S_{\text {irr }}$ versus $\lambda$ for $1 \mathrm{D}$ and $2 \mathrm{D}$ Dirac Hamiltonians with different $\beta$ and $\delta=0.01$. There is a sharp peak at $\lambda=0$ for $1 \mathrm{D}$ which gets broadened in the $2 \mathrm{D}$ case. Here, $\beta$ increases from top to bottom.
1 P. Talker, E. Lutz and P. Hanggi, Phys. Rev. E 75, 050102 (R) (2007);

2 P. Talker and P. Hanggi J. Phys. A 40, F569 (2007).

3 S. Deffner and E. Lutz, Phys. Rev. Lett. 105, 170402 (2010)
${ }^{4}$ R. Dorner, J. Goold, C. Cormick, M. Paternostro and V. Vedral, Phys. Rev. Lett. 109, 160601 (2012).

${ }^{5}$ G. E. Crooks, Phys. Rev. E 60, 2721 (1999).

${ }^{6}$ H. Tasaki, arXiv:cond-mat/00079244v2 (2000).

7 C. Jarzynski, Phys. Rev. Lett. 78, 2690 (1997). 
8 S. Sotiriadis, A. Gambassi, and A. Silva, Phys. Rev. E 87, 052129 (2013).

9 P. Zanardi, L. C. Venuti and P. Giorda, Phys. Rev. A 76, 062318 (2007).

10 S. Deffner and E. Lutz, Phys. Rev. E 87, 022143 (2013).

11 R. Dorner, S. R. Clark, L. Heaney, R. Fazio. J. Goold and V. Vedral, Phys. Rev. Lett. 110, 230601 (2013).

12 S. Deffner and E. Lutz, Phys. Rev. Lett. 107, 140404 (2014).

13 F. Plastina, A. Alecce, T. J. G. Apollaro, G. Falcone, G. Francica, F. Galve, N Lo Gullo and R. Zambrini, Phys. Rev. Lett., 113, 260601 (2014).

14 S. Sachdev, Quantum Phase Transitions (Cambridge University Press, Cambridge, UK, 1999).

15 S. Suzuki, J-i Inoue and Bikas K. Chkarabarti, Quantum Ising Phases and Transitions in Transverse Ising Models (Springer, Lecture Notes in Physics, Vol. 862 (2013)).

16 A. Dutta, U. Divakaran, D. Sen, B. K. Chakrabarti, T. F. Rosenbaum and Gabriel Aeppli arXiv:1012.0653 2 (2010).

17 P. Zanardi. H. T. Quan, X. Wang and C. P. Sun, Phys. Rev. A 75, 032109 (2007).

18 H.T. Quan, Z. Song, X.F. Liu, P. Zanardi, and C.P. Sun, Phys.Rev.Lett. 96, 140604 (2006).

19 D. Rossini, T. Calarco, V. Giovannetti, S. Montangero, R. Fazio, Phys. Rev. A 75, 032333 (2007).

20 S. Sharma, V. Mukherjee, and A. Dutta, Eur. Phys. J. B, 85, 143 (2012).

21 V. Mukherjee, S. Sharma, A. Dutta, Phys. Rev. B 86, 020301 (R) (2012).

22 T. Nag, U. Divakaran and A. Dutta, Phys. Rev. B 86, 020401 (R) (2012).

23 M. Heyl, A. Polkovnikov, and S. Kehrein, Phys. Rev. Lett., 110, 135704 (2013).

24 A. Silva, Phys. Rev. Lett. 101, 120603 (2008).

25 A. Gambassi and A. Silva, arXiv:1106.2671 (2011).

26 P. Smacchia and A. Silva, Phys. Rev. E 88, 042109 (2013).

27 C. De Grandi, V. Gritsev, and A. Polkovnikov, Phys. Rev. B 81, 012303 (2010).

28 A. Polkovnikov, K. Sengupta, A. Silva, and M. Vengalattore, Rev. Mod. Phys. 83 ,863 (2011).

29 J. Dziarmaga, Advances in Physics (2011).

30 E. Mascarenhas, H. Braganca, R. Dorner, M. Franca San- tos, V. Vedral, K. Modi, and J. Goold, Phys. Rev. E 89, 062103 (2014).

31 Zanardi P. and Paunkovic N., Phys. Rev. E, 74 (2006) 031123.

32 Campos Venuti L. and Zanardi P., Phys. Rev. Lett., 99 (2007) 095701; Zanardi P., Giorda P. and Cozzini M., Phys. Rev. Lett., 99 (2007) 100603.

33 S-J Gu, Int. J. Mod. Phys. B 24, 4371(2010).

34 M. M. Rams and B. Damski, Phys. Rev. Lett. 106 , 055701 (2011).

35 M. E. Fisher and Michael N. Barber Phys. Rev. Lett. 28, 1516 (1972); M. N. Barber, in Phase Transitions and Critical Phenomena Vol. 8. C. Domb, , and J. L. Lebowitch, (eds) New York: Academic Press (1983).

36 M. M. Rams and B. Damski, Phys. Rev. A 84, 032324 (2011).

37 V. Gritsev and A. Polkovnikov, in Understanding Quantum Phase Transitions, edited by L. D. Carr (Taylor and Francis, Boca Raton) 2010; arXiv:0910.3692 (2009).

38 D. Schwandt, F. Alet and S. Capponi, Phys. Rev. Lett., 103 (2009) 170501; A. A. Albuquerque F. Alet, C. Sire and S. Capponi, Phys. Rev. B, 81 (2010) 064418.

39 V. Mukherjee, A. Polkovnikov and A. Dutta, Phys. Rev. B, 83 (2011) 075118.

40 A. H. C. Neto, F. Guinea, N. M. R.Peres, K. S. Novoselov and A. K. Geim Rev. Mod. Phys. 81, 109 (2009).

41 M. Z. Hasan and C. L. Kane, Rev. Mod. Phys. 82 3045(2010).

42 X.-L. Qi and S. -C. Zhang, Rev. Mod. Phys.83, 1057 (2011).

43 A. Dutta, R. R. P. Singh and U. Divakaran, EPL 89, 67001 (2010).

44 V. Mukherjee, and A. Dutta, Phys. Rev. B 83, 214302 (2011).

45 A. A. Patel and A. Dutta, Phys. Rev. B 86, 174306 (2012).

46 V. Mukherjee, A. Dutta and D. Sen, Phys. Rev. B, 85 (2012) 024301.

47 A. A. Patel, S. Sharma and A. Dutta , EPL, 102, 46001, (2013).

48 S. Sharma, A. Russomanno, G. E. Santoro and A. Dutta, EPL 106, 67003, (2014). 